\title{
Ising Meets Ornstein and Zernike, Debye and Hückel, Widom and Rowlinson, and Others
}

\author{
Ronald Dickman* \\ Departamento de Física, ICEx, \\ Universidade Federal de Minas Gerais, \\ 30123-970 Belo Horizonte - MG, Brasil
}

Received on 20 August, 2000

\begin{abstract}
The name Ising has come to stand not only for a specific model, but for an entire universality class - arguably the most important such class - in the theory of critical phenomena. I review several examples, both in and out of equilibrium, in which Ising universality appears or is pertinent. The "Ornstein-Zernike" connection concerns a thermodynamically self-consistent closure of the eponymous relation, which lies at the basis of the modern theory of liquids, as applied to the Ising lattice gas. Debye and Hückel founded the statistical mechanics of ionic solutions, which, despite the long-range nature of the interaction, now appear to exhibit Ising-like criticality. The model of Widom and Rowlinson involves only excluded-volume interactions between unlike species, but again belongs to the Ising universality class. Far-from-equilibrium models of voting behavior, catalysis, and hysteresis provide further examples of this ubiquitous universality class.
\end{abstract}

\section{Introduction}

Since the playful title of this review might generate confusion, let me start by saying that I have no idea whether or not Ernst Ising actually met any of the other scientists mentioned. The world lines intersecting here belong to models and theories, not to persons, real or imagined! Much as the name Galileo has come to define a specific kind of relativity, Hamilton a kind of dynamics, and Gauss a distribution, so "Ising" is now indelibly associated with a specific kind of critical behavior, a "universality class" in the familiar jargon of renormalization group theory. The latter tells us (as is borne out by experiment, analysis, and simulation), that the factors determining scaling properties in the neighborhood of the critical point are few, and pertain to very general properties of the system, such as its dimensionality and that of the order parameter, range and symmetry of interactions. For historical reasons, the class defined by a scalar order parameter, $\phi$, short-range interactions, isotropy, and symmetry under inversion $(\phi \rightarrow-\phi$ in the absence of an external field), is commonly known as the Ising universality class. While we should expect many systems to fall in this class, most of the present article is devoted to examples whose membership is surprising or controversial.

My first example, self-consistent Ornstein-Zernike theory (Sec. II), is not a bona-fide member of the Ising class, but is, in a sense, making an impressive effort to be one! Criticality in electrolyte solutions, discussed in Sec. III, remains controversial, though recent theoretical and experimental studies support Ising-like behavior. We will see that the simplest lattice version of the well-known primitive model may exhibit a line of Isinglike critical points. We are used to thinking of the Ising model as possessing two relevant variables (or scaling fields), the temperature and the external magnetic field. The Widom-Rowlinson model (Sec. IV) (in its latticegas version) illustrates the possibility of Ising criticality in a system with no temperature to speak of. Having dispensed with temperature, we proceed in Sec. V to the more radical step of eliminating the Hamiltonian (and thermodynamics) altogether, in several far-fromequilibrium stochastic processes. The guiding principle of symmetry again permits one to understand the appearence of Ising criticality in these systems. A brief summary is provided in Sec. VI.

\section{Self-Consistent Ornstein- Zernike Theory for the Ising Model}

Ornstein-Zernike theory [1] represents an important, early chapter in the theory of critical phenomena, and is still widely used to interpret data from scattering experiments. The Ornstein-Zernike relation (OZR), moreover, serves as the starting point for modern theories of

\footnotetext{
*email: dickman@fisica.ufmg.br
} 
liquids [2]. This relation introduces the direct correlation function $c(r)$ [4] in terms of the total correlation function $h(r) \equiv g(r)-1$, where $g(r)$ is the radial distribution function:

$$
h(r)=c(r)+\rho \int d \mathbf{r}^{\prime} c(r) h\left(\left|\mathbf{r}-\mathbf{r}^{\prime}\right|\right) .
$$

Here $\rho$ is the number-density. Of course one must now supply a closure, i.e., a second relation between $c(r)$, the intermolecular potential $w(r)$, and (in certain cases) $h(r)$ itself. This, in general, can be done only in a heuristic manner, as for example in the mean-spherical approximation, $c(r)=-w(r) / k_{B} T$, or the more sophisticated Percus-Yevick or hypernetted chain closures $[2,3]$. Surprisingly, these simple closures lead in many cases to good predictions for $h(r)$, and for thermodynamic properties, via one of the standard, exact relations (the so-called virial, compressibility, and energy routes) involving $h(r)$. This is remarkable, since neither the OZR nor the closure include any thermodynamic input. The $\mathrm{OZ}$ formalism and the resulting 'integral equations' for $h(r)$ are couched purely in terms of correlations, without reference to a free energy.

The absence of thermodynamic input becomes evident when one compares the predictions from different routes. Thus the Percus-Yevick closure yields two different equations of state for the hard-sphere fluid, depending on whether the link to thermodynamics is made using the virial or the compressibility route. Such inconsistencies suggest a new strategy for closing the OZR, in which we use a thermodynamic relation, expressing equality of (for example) the pressure evaluated via different routes, to find a second relation between $h, c$, and $w$. Such an approach was proposed over twenty years ago by Høye and Stell [5], but detailed numerical implimentations have appeared only in the last few years. The first such application was, naturally, to the three-dimensional Ising model, which we outline here. (For details the interested reader may consult Refs. [6] and [7].)

We consider the Ising lattice gas with independent variables $\rho$ (number of particles per site) and $\beta \equiv 1 / k_{B} T$. The interparticle potential $w(r)$ is zero for $r>1,-1$ for $r=1$ (an attractive nearest-neighbor interaction) and infinite for $r=0$. The latter implies the 'core-condition' $h(0)=-1$, expressing the simple fact that two particles may not occupy the same site. On a lattice the virial route, which involves the spatial derivative of the potential, does not exist. Consistency between the two remaining routes, energy and compressibility, is embodied in the relation

$$
\rho \frac{\partial^{2}(\rho u)}{\partial \rho^{2}}=\frac{\partial^{2}(\beta p)}{\partial \beta \partial \rho}
$$

where $u$ is the internal energy per particle and $p$ is the pressure. The energy relation is

$$
u=-\frac{1}{2} q \rho\left(1+h_{1}\right),
$$

where $h_{1}$ denotes the total correlation function at the nearest-neighbor separation, and $q$ is the coordination number. The inverse compressibility is related to $c$ via

$$
\frac{\partial(\beta p)}{\partial \rho}=1-\rho \tilde{c}(0)
$$

where $\tilde{c}$ denotes the Fourier transform. The direct correlation function is, in effect, defined by the OZR, which reads, on lattice,

$$
h_{\mathbf{i}}=c_{\mathbf{i}}+\rho \sum_{\mathbf{j} \in \mathcal{L}} c_{\mathbf{i}-\mathbf{j}} h_{\mathbf{j}},
$$

(the sum is over the sites of lattice $\mathcal{L}$ ). This relation, or, more specifically, its Fourier representation,

$$
1+\rho \tilde{h}=\frac{1}{1-\rho \tilde{c}},
$$

enables us to find $h$ given $c$. Since we already have $h(0)=-1$, the above relations, incorporating thermodynamic consistency, suffice for determining one free parameter, as a function of $\rho$ and $\beta$. At this stage we introduce the unique approximation of our theory, which is to set $c(r) \equiv 0$ for $r>1$, i.e., beyond the range of the interaction. This defines what we call the selfconsistent Ornstein-Zernike approximation or SCOZA. After some manipulations we obtain a nonlinear partial differential equation for $c(1)=c_{1}(\beta, \rho)(c(0)$ is effectively fixed by the core condition). Integrating this PDE numerically eventually yields $h(r)$ and all thermodynamic properties of the lattice gas.

The results compare remarkably well against the best numerical (series expansion) estimates for the lattice gas $[6,7]$. We find the critical temperature from the condition of a diverging compressibility at $\rho=1 / 2$ (below $T_{c}$, infinite compressibility signals the spinodal); the coexistence curve is constructed in the usual manner. Especially impressive are the predictions for critical parameters, (Ref.[6]); SCOZA reproduces the best series estimates to within $0.2 \%$.

Can SCOZA predict critical exponents? Its thermodynamic predictions are so accurate that effective exponents (i.e., derivatives of the compressibility, order parameter, etc., with respect to temperature) are in reasonable agreement with Ising model values near, but not asymptotically close to, the critical point. We really should not expect SCOZA to reproduce Ising critical exponents: $c(r)$ is not zero for $r>1$ in the Ising model. In particular, it develops a power-law tail at the critical point. The actual critical behavior of the SCOZA-lattice gas is unusual [7]: for $T>T_{c}$ the exponents are those of the spherical model: $\gamma=2, \delta=5$, and $\alpha=-1$ (the specific heat does not diverge at $T_{c}$ ), as compared with $\gamma \simeq 1.24, \delta \simeq 4.8$, and $\alpha \simeq 0.1$ for 
the three-dimensional Ising model. Below $T_{c}$, SCOZA yields a new set of exponents: $\gamma^{\prime}=7 / 5, \beta^{\prime}=7 / 20$ and $\alpha^{\prime}=-1 / 10$, considerably better than mean-field or spherical model values.

Clearly the way to improve SCOZA is to relax the truncation of $c(r)$. But even with its current limitations, SCOZA represents the first theory to yield globally accurate thermodynamic and structural properties for a fluid model. It has been applied with success to off-lattice fluids [11], and fluids in a disordered matrix [12], and promisses to become an important tool in the study of liquids.

\section{Ising Universality and the Primitive Model of Elec- trolytes}

The critical behavior of electrolyte solutions remains a challenging area, both experimentally and theoretically. Experiments on various ionic solutions have yielded either mean-field like critical exponents [13], Ising-like exponents [14], or a crossover from mean-field to Isinglike behavior as one nears the critical temperature [15]. In some cases the crossover from mean-field to Ising exponents occurs at a reduced temperature much smaller than that typically seen in non-Coulomb liquids.

Theoretical studies have tended to focus on the simple system introduced by Debye and Hückel in 1923 [16], commonly known as the restricted primitive model (RPM). Here the positive and negative ions are represented by hard spheres of diameter $\sigma$, containing point charges $\pm q$ at their centers. The solvent is not considered explicitly; its effect is represented solely by the dielectric constant $\epsilon$ that enters the expression for the electrostatic potential. While clearly a minimal model for ionic solutions, the RPM already presents great difficulty to theory and simulation. Debye and Hückel's analysis of the dilute, high-temperature regime led to the fundamental result that electrostatic interactions are screened on a length scale $\Gamma^{-1}$, where $\Gamma^{2}=4 \pi \rho \beta q^{2} / \epsilon$. (The effective interaction or potential of mean force takes on a Yukawa form $\propto e^{-r / \Gamma} / r$.)

What sort of phase diagram should we expect for the RPM? The hard-sphere part of the potential alone will lead to a fluid-solid transition. At high temperatures $\left(T^{*} \equiv \sigma k_{B} T \epsilon / q^{2} \gg 1\right)$ we expect packing considerations to dominate, so that the high-density structure is FCC or HCP. But since these structures are incompatible with antiferromagnetic order (i.e., positive and negative charges occupying distinct sublattices), we should expect a structural phase transition to a bipartite lattice (presumably BCC) as we lower the temperature, at high density.

The opposite corner of the $\rho-T$ plane, low temperature and density much less than the solid, is where we find the critical point in simple fluids. Since the RPM lacks the short-range attraction that drives the liquid-gas transition, it is not immediately obvious that it should exhibit such a transition. Nevertheless, Stell, $\mathrm{Wu}$, and Larsen, using liquid-state theory, reached the conclusion that the RPM has a liquid-gas coexistence curve with a critical point [17], but with a critical density much lower than for a simple argon-like fluid. Monte Carlo simulations confirm these conclusions, but only in the last few years have the studies of different groups converged toward common values for the critical density and temperature of the RPM [18-20]. Given the difficulty in simply locating the critical point, it is not surprising that current simulations shed little light on its nature.

On the theoretical side, however, there has been considerable discussion of RPM criticality, pointing toward Ising-like behavior [21-23]. This seems at odds with conventional wisdom (Ising universality for shortrange, and mean-field behavior for long-range interactions), but may be understood intuitively as follows. At the low temperatures of interest (note that $T_{c}^{*} \approx 0.05$ ), the Coulombic interaction strongly suppresses chargedensity fluctuations on scales larger than $\sigma$; ions associate into pairs and larger aggregates. Thus the effective interacting units are not individual ions but clusters that are typically neutral (or nearly so), interacting via multipolar forces (presumably quadrupole and higher) that are of short range and, on average, attractive (since such fluctuations lower the energy).

Such an intuitive picture finds support in recent experiments showing Ising critical exponents at an ionicsolution critical point [15]. What is needed, from the theoretical standpoint, is an argument that takes us from the RPM to a continuum description of density fluctuations, $\delta \rho(\mathbf{r})$, having the same form (up to irrelevant terms) as that for the Ising model, i.e., the usual $\phi^{4}$ field theory. It is clear, on the other hand, that the RPM needs to be described in terms of a pair of coupled fields, the mass or number density $\delta \rho$ and the charge density $\psi$. An important first step is the proper formulation of a mean-field theory $[24,25]$. Very recently, Ciach and Stell constructed a Landau-Ginzburg free energy functional, starting from the mean-field theory of the RPM, in terms of the two fields, $\delta \rho$ and $\psi$. They find that integrating out the charge density fluctuations leads to an effective field theory for $\delta \rho$ having the expected $\phi^{4}$ form. (Essentially, an attractive effective interaction between mass-density fluctuations is mediated by charge-charge correlations.) Thus the crucial link between the RPM and Ising-like criticality appears to be at hand.

In light of the great difficulty of RPM simulations, it seems useful to study a lattice restricted primitive model (LRPM) [26, 27], since lattice simulations offer substantial economies in the computation time required for evaluating the potential. (Overlap checks are triv- 
ial using a site-occupancy matrix, while the Coulomb potential - with the contributions from the infinite periodic array of cells suitably accounted for - may be stored in a lookup table.) Dickman and Stell [26] considered a lattice gas of particles interacting via site exclusion (multiple occupancy forbidden) and a Coulomb interaction $u\left(r_{i j}\right)=s_{i} s_{j} / r_{i j}$, where $r_{i j}=\left|\mathbf{r}_{i}-\mathbf{r}_{j}\right|$ is the distance separating the particles (located at lattice sites $\mathbf{r}_{i}$ and $\mathbf{r}_{j}$ ), and $s_{i}=+1$ or -1 is the charge of particle $i$. (Exactly half the particles are positively charged, half negative. They are restricted to a simple cubic lattice with periodic boundaries.)

At full occupancy, the LRPM may be viewed as an antiferromagnetic Ising model with long-range interactions. One naturally expects a critical or Néel point separating a high-temperature phase from a phase exhibiting antiferromagnetic order. (The Ising analogy facilitates formulation of a mean-field theory for the LRPM.) Indeed, it was proven some time ago that the LRPM on the simple cubic lattice exhibits long-range order at sufficiently low temperatures and high fugacities [28]. In simulations, we find that this point marks one terminus of a line of critical points in the $\rho-T$ plane (see Fig. 1). The other end of the critical line is a tricritical point, which intersects the coexistence curve between a low-density disordered phase and the ordered phase. The Monte Carlo simulations used a relatively small lattice $\left(16^{3}\right.$ sites $)$, sufficient to map out the phase diagram by studying the order parameter, specific heat, and correlation functions, but too small to yield information on critical behavior [26].

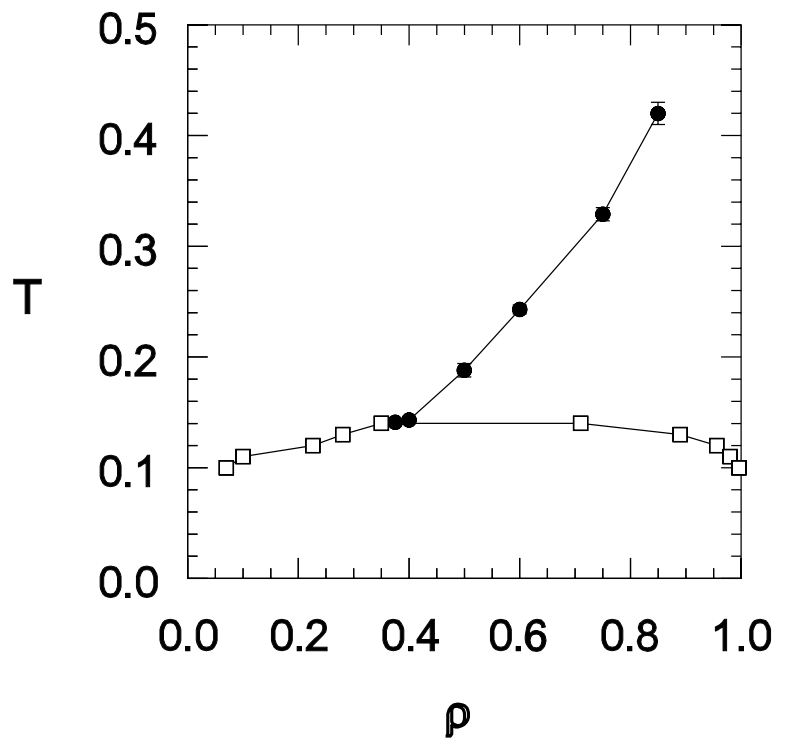

Figure 1. Best estimates for the location of the critical line and coexistence curve in the lattice RPM (simple cubic lattice).

Thus the simplest version of the LRPM shows a phase diagram that is rather different than the continuous-space RPM, exhibiting a critical line and tricritical point rather than a simple critical point. One reason for this difference is that the simple cubic lattice facilitates antiferromagnetic order. It may be that on a lattice that frustrates such order (the FCC structure, for example), the critical line will disappear, and the tricritical point become a critical point. On the other hand, Panagiotopoulos and Kumar found exactly this, on the simple cubic lattice, when the hard-core exclusion range is $\geq 3$ times the lattice spacing [27]. A suitably modified version of the LRPM may therefore be useful for studying ionic criticality. The phase diagram appears to be highly sensitive to changes in lattice structure or short-range interactions, as was also noted by Ciach and Stell [25].

Even if the critical line of the simple-cubic LRPM is not exactly what we had in mind for understanding the off-lattice RPM, its nature, and that of the associated tricritical point, are of interest, and potentially relevant to other systems with Coulombic interactions. Given the symmetry of the system, the Ising universality class again seems the natural candidate. The (very limited) simulation data seem to indicate $\beta \leq 0.326$, (the 3 -d Ising value), which should in any case rule out a mean-field type transition. Clearly larger-scale simulations and finite-size scaling analysis are in order; a low-temperature expansion for the fully occupied case might also prove useful. We close this section with the observation that Ising criticality is compatible with a long-range bare interaction, provided the effective interactions between critical fluctuations are of short range.

\section{Ising Without Temperature: The Widom-Rowlinson Model}

In this section we again consider a model imported to the lattice from its original continuous-space formulation. The Widom-Rowlinson (WR) hard-sphere mixture is perhaps the simplest binary fluid model showing a continuous unmixing transition, and has been the subject of considerable study regarding its thermal and interfacial properties $[29,30]$, as has the Gaussian $f$ function version of the model introduced somewhat earlier by Helfand and Stillinger [31]. Despite this interest, however, definitive results on the location and nature of the WR critical point are lacking. As a first step in this direction, Dickman and Stell performed extensive simulations of the lattice-gas analog of the WR hard-sphere mixture the Widom-Rowlinson lattice model (WRL) [32].

In the original WR model, $\mathrm{AB}$ pairs interact via a hard-sphere potential whilst AA and BB pairs are noninteracting [29]. The WRL model is a two-component lattice gas in which sites may be at most singly oc- 
cupied, and in which nearest-neighbor A-B pairs are forbidden. Like the WR model, this is evidently an athermal model (all allowed configurations are of the same energy), and is characterized solely by the densities of the two species or by the corresponding chemical potentials $\mu_{A}$ and $\mu_{B}$. $\left(\mu_{A}=\mu_{B}=\mu_{c}\right.$ of course, at the critical point.)

Despite the absence of a temperature or energy scale, the WRL model has a close affinity to the Ising model. To see this, note that the WRL may be viewed as an extreme member of a family of binary alloy models with nearest-neighbor interactions that are repulsive between unlike species (interaction energies $\epsilon_{A B}>0$, $\left.\epsilon_{A A}=\epsilon_{B B}=0\right)$. The Ising model may be transcribed into such a model by identifying up and down spins with A and B particles, respectively, yielding a "closepacked" alloy that unmixes at the Ising critical temperature. Allowing a small fraction of vacant sites results in a dilute binary alloy (DBA) with a somewhat depressed critical temperature; continuing the dilution process, one arrives at a model with $T_{c}=0$. This zerotemperature terminus of the DBA critical line is precisely the WRL critical point. The coexistence surface of the binary alloy model in the $\rho_{A}, \rho_{B}, T$ solid is shown schematically in Fig. 2. One is then led to ask whether the entire critical line shares a common behavior, or whether its character changes at some point. Although the former is clearly favored on the basis of universality, a careful examination of this question nevertheless appears worthwhile.

\section{BINARY ALLOY COEXISTENCE SURFACE}

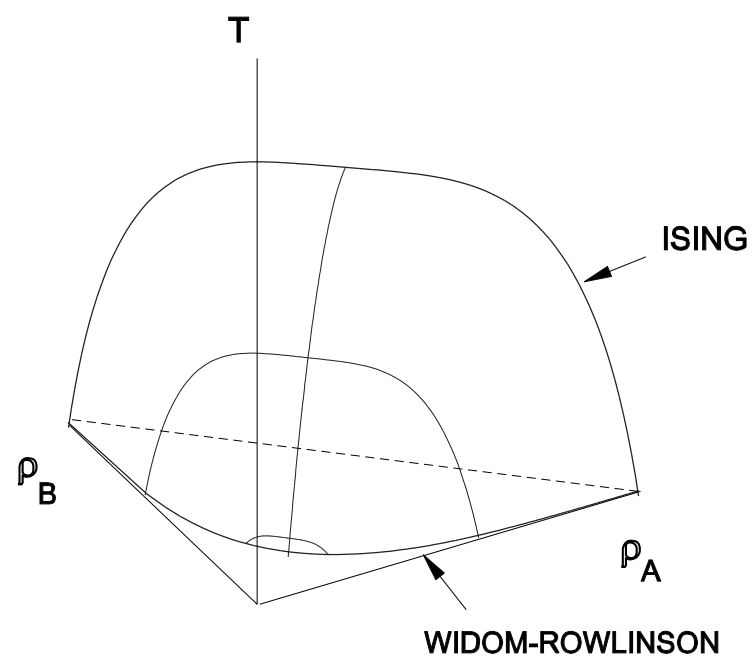

Figure 2. Schematic coexistence surface of the binary alloy model. The Ising model coexistence curve is the intersection of the coexistence surface with the plane $\rho_{A}+\rho_{B}=1$. The WRL coexistence curve is the intersection with the plane $T=0$.

As shown in Fig. 2, the Ising coexistence curve is the intersection of the critical surface with the plane $\rho \equiv \rho_{A}+\rho_{B}=1$. For $\rho<1$ we can cross the critical line in the temperature or the density direction, maintaining all the while $h \equiv \mu_{A}-\mu_{B}=0$ ( $\mu_{i}$ is the chemical potential of species $i$ ). Thus, while in the WRL there is no temperature per se, $\mu \equiv \mu_{A}+\mu_{B}$ is a temperaturelike variable, so that along the symmetry line $h=0$, and in the vicinity of the critical point $\mu_{c}$, the susceptibility should scale as $\chi \simeq\left(\mu-\mu_{c}\right)^{-\gamma}$, the order parameter, $\rho_{A}-\rho_{B} \simeq\left(\mu-\mu_{c}\right)^{\beta}$, (for $\left.\mu>\mu_{c}\right)$, and so on [31]. (Note that if the chemical potentials are taken as independent variables, no "Fisher renormalization" of critical exponents is expected, as it would be if we worked with fixed densities [33].)

Ref. [32] reports Monte Carlo simulations of the WRL in the grand canonical ensemble, using lattices of up to $160^{2}$ sites in two dimensions and $64^{3}$ sites in three dimensions. The algorithm employs three kinds of moves: "flips" (change of the state, A, B, or vacant, at a single site), "exchanges" (between any pair of sites in the system), and flips $(A \rightleftharpoons B)$ of entire clusters (note that this is always possible in the WRL since a cluster of occupied sites is always surrounded by a border of vacant sites). The results for the reduced fourth cumulant, order parameter, and susceptibility are all consistent with Ising-like behavior [32].

Following the WRL study, a series analysis of a closely related continuum model, with Gaussian Mayer $f$-functions, (i.e., the model introduced in Ref. [31]), was reported by Lai and Fisher [34]. This study again provides good evidence for consistency with the Ising universality class at the critical point marking phase separation. More recently, the generalization of the WRL to $q$ states, with infinite repulsion between unlike nearest-neighbor pairs has been found to exhibit critical behavior consistent with the $q$-state Potts model, as would be expected from symmetry considerations [35].

The appearance of Ising-like criticality in athermal models in fact goes back to studies of lattice gases with nearest-neighbor exclusion (NNE), done in the mid1960 's [36-38]. In this case there is only a single species of particle, and the only interaction is a hard-core repulsion assigning infinite energy to pairs of particles with separations $\leq 1$, in units of the lattice spacing. On a bipartite lattice in two or more dimensions, there is a critical density above which the particles begin to occupy one of the two sublattices preferentially, signalling a continuous transition to a state with antiferromagnetic order. (The NNE lattice gas is in fact closely related to the zero-temperature line of the Ising antiferromagnet.) Gaunt and Fisher analyzed series expansions for the NNE lattice gas on various two- and three-dimensional lattices, and found $\beta \approx 1 / 8$, suggesting, once again, Ising universality [36, 37]. It would be worthwhile applying modern series and simulation methods to the NNE models, to obtain more precise results on their critical behavior.

It is perhaps worth observing that in all of the mod- 
els mentioned in the present section (and, indeed, in the hard-sphere fluid), phase separation is driven exclusively by entropy maximization. Thus we have the apparently paradoxical conclusion that for large values of the chemical potential, the ordered phase has an entropy greater than or equal to that of the disordered phase. This shows that the naive identification of entropy with 'disorder' is not always appropriate; interpreting increased entropy as greater freedom seems more apt.

\section{Ising Without Equilibrium: Voters, Catalysis, and Hys- teresis}

The present section concerns far-from-equilibrium models, defined by a Markovian dynamics rather than a Hamiltonian. Since the transition rates do not satisfy detailed balance with respect to any reasonable energy function (i.e., bounded below, and with a finite number of many-body terms), these models have no thermodynamic interpretation. Such systems are nevertheless widely studied using the tools of statistical physics, as models of, for example, populations, traffic, catalysis, and "self-organized" criticality [40]. Many of these systems exhibit transitions between a fluctuation-free absorbing state (admitting no escape) and an active phase [41, 42, 43]. Models possessing an absorbing state, and, associated with this, a non-negative order-parameter density, do not exhibit Ising symmetry. Instead, they fall generically in the universality class of directed percolation, which plays a role analogous to the Ising class for absorbing-state phase transitions [39, 44].

The models to be discussed in this section do, however, observe Ising symmetry, and belong to the Ising universality class. The fact that the Ising class cuts across the equilibrium/nonequilibrium boundary serves to illustrate that phase transitions in far-fromequilibrium systems are just as worthy of the name as their equilibrium counterparts, and do not, as is sometimes asserted, represent merely an "analogy" to "real" (i.e., equilibrium) phase transitions. Of course, one's outlook will depend on whether one adopts a thermodynamic or a mathematical definition of a phase transition. My point is that experience with percolation, nonequilibrium models, and deterministic as well as stochastic cellular automata make it natural to regard, quite generally, any singular dependence of the properties of a system of many interacting units upon its control parameters as a phase transition. From this vantage, critical phenomena represent a particular class of singularities (attended, for example, by a diverging correlation length and relaxation time), with equilibrium critical points representing a particular, and not especially privileged, subset thereof.

\section{The Majority-Vote Model}

The prototypical example of a nonequilibrium stochastic process with local interactions, isotropy, and updown symmetry is the majority-vote model [44]. At each site of a lattice there is a "spin" variable $\sigma_{i}= \pm 1$. A Markov process is defined as follows. At each step, a site $i$ is chosen at random, and $\sigma_{i} \rightarrow \sigma_{i}^{\prime}$, which is taken to be equal to the majority of its nearest neighbors (i.e., $\operatorname{sgn}\left[\sum_{j n n i} \sigma_{j}\right]$ ) with probability $p$, and to the minority with probability $q=1-p$. (If there is no majority $\sigma_{i}$ flips with probability $1 / 2$.) Although the transition rates do not satisfy detailed balance, it is not hard to see that the parameter $q$ plays a role analogous temperature in the equilibrium kinetic Ising model. (The dynamics of the majority vote model is akin to Glauber dynamics; there are no conserved quantities.) For $q=0$, "voters" have no independence of opinion whatever, and slavishly follow the local majority. This $q=0$ limit defines the voter model, which has two absorbing states, all +1 or all -1 , just as in the Ising model at $T=0$. (The voter model belongs to a universality class different than Ising, the so-called compact directed percolation class $[45,46]$.) For $q>0$ but small, the stationary state exhibits spontaneous "magnetization": $m \equiv\left\langle\sigma_{i}\right\rangle= \pm m_{0}$, with $m_{0}>0$. Above a critical $q$ value $\left(q_{c} \simeq 0.075\right.$ for the square lattice [47]), the stationary state is disordered $(m=0)$. (No ordered state is found in one dimension, just as for the equilibrium Ising model.)

Thus the phase diagram of the majority-vote model is qualitatively the same as for the standard Ising model. Confirmation that the critical behavior is also of the Ising kind comes from Monte Carlo simulations reported by de Oliveira [47], which showed (for the twodimensional case), that the exponents $\nu, \gamma$ and $\beta$ are the same as those of the Ising model. Many nonequilibrium models have now been shown to exhibit Ising-like critical behavior, indicating that this universality class is equally robust, in or out of equilibrium. Examples include a family of generalized majority-vote models [48], an anisotropic majority-vote model [49], a two-state immunological model [50], and various two-temperature Ising models [51]. In the latter example, a spin system evolves via two dynamical processes, for example, single-spin flips and nearest-neighbor exchanges, each having its own temperature (see Ch. 8 of Ref. [43] for a review). Ising-like short-time critical behavior has also been established for certain nonequilibrium models with up-down symmetry [52].

The principle underlying universality of critical behavior in these examples appears, once again, to be symmetry. While detailed renormalization group analyses are lacking, Grinstein et al. have argued that models obeying up-down symmetry and exhibiting a continuous phase transition, be they in equilibrium or not, will have a coarse-grained descrption of the same form as for 
a standard kinetic Ising model [53]. We can see this as follows: if such a description exists, then in a Langevinlike equation for the order parameter density $m(\mathbf{r}, t)$, only odd powers of $m$ may appear (up-down symmetry). Gradient terms are ruled out by isotropy, so the lowest-order derivative term will be $\nabla^{2} m$. Generically, the transition to an ordered state will be controlled by the coefficient $a$ of the term $\propto m$. (In mean-field theory this coefficient vanishes at the critical point, but in a full analysis the nonlinear terms will renormalize $a_{c}$ to a nonzero value.) That is, the order-parameter density will obey (in the nonconserving case) a time-dependent Landau-Ginzburg equation of the form

$$
\frac{\partial m(\mathbf{r}, t)}{\partial t}=\nabla^{2} m-a m-b m^{3}+\eta(\mathbf{r}, t)
$$

where we have dropped higher-order terms ( $\propto m^{5}$, etc.) since they are irrelevant to critical behavior. The noise term $\eta(\mathbf{r}, t)$ is zero-mean, and Gaussian, with autocorrelation $\left\langle\eta(\mathbf{r}, t) \eta\left(\mathbf{r}^{\prime}, t^{\prime}\right)=\Gamma \delta^{d}\left(\mathbf{r}-\mathbf{r}^{\prime}\right) \delta\left(t-t^{\prime}\right)\right.$. In equilibrium $\Gamma$ is of course proportional to temperature. Out of equilibrium no such relation exists, but the theory again predicts an Ising-like transition; the deviation from equilibrium is found to be irrelevant, near the upper critical dimension [53]. The principle that systems with the same symmetries share a common critical behavior, whether in or out of equilibrium, has recently been extended to models with Potts-like symmetries $[54,55]$.

We have seen that a variety of nonequilibrium perturbations preserve the character of the transition in spin systems or lattice gases. It is worth noting that biasing the hopping rates to favor movement along a particular axis (e.g., in a lattice gas with attractive interactions and a Kawasaki-type exchange dynamics) breaks an essential symmetry of the Ising model by introducing a preferred direction. Such "driven diffusive systems", as they have come to be called, exhibit critical behavior outside the Ising class. The precise nature of the transition remains controversial; for reviews see Refs. [43] and [56].

\section{Catalysis Models}

Another large class of nonequilibrium lattice models arises in the study of heterogeneous catalysis, typically on a metallic surface. One of the first such models to be studied in detail was introduced by Ziff, Gulari and Barshad (ZGB), to describe the reaction $\mathrm{CO}+1 / 2 \mathrm{O}_{2}$ $\rightarrow \mathrm{CO}$ on a platinum surface [57]. These models typically exhibit transitions to an absorbing state, which (when continuous) fall in the directed percolation class [58]; they are reviewed in chapter 5 of Ref. [43].

The ZGB model exhibits two phase transitions, one continuous, the other discontinuous. Under a suitable perturbation, however, the latter can become a critical point, which appears to belong to the Ising class. In the ZGB model, the catalytic surface is represented by a two dimensional lattice. The transition rates involve a single parameter, $Y$ : the probability that a molecule arriving at the surface is CO. This molecule needs only a single vacant site to adsorb, as indicated by experimental studies; $\mathrm{O}_{2}$ requires a pair of vacant sites. After each adsorption event, the surface is immediately cleared of any $\mathrm{CO}-\mathrm{O}$ nearest-neighbor (NN) pairs, making the reaction rate for $\mathrm{CO}_{2}$ formation, in effect, infinite. The adsorption and reaction events comprising the dynamics go as follows. First choose the adsorbing species - CO with probability $Y, \mathrm{O}_{2}$ with probability $1-Y$ - and a lattice site $\mathbf{x}$ (or, in the case of $\mathrm{O}_{2}$, a $\mathrm{NN}$ pair, $(\mathbf{x}, \mathbf{y})$ ), at random. If $\mathbf{x}$ is occupied (for $\mathrm{O}_{2}$, if $\mathbf{x}$ and/or $\mathbf{y}$ are occupied), the adsorption attempt fails. If the newly-adsorbed molecule is CO, determine the set $\mathcal{O}(\mathbf{x})$ of NNs of $\mathbf{x}$ harboring an $\mathrm{O}$ atom. If this set is empty, the newly-arrived CO remains at $\mathbf{x}$; otherwise it reacts, vacating $\mathbf{x}$ and one of the sites in $\mathcal{O}(\mathbf{x})$, (chosen at random if $\mathcal{O}(\mathbf{x})$ contains more than one site). If the newly-adsorbed molecule is $\mathrm{O}_{2}$, construct $\mathcal{C}(\mathbf{x})$, the set of neighbors of $\mathbf{x}$ harboring $\mathrm{CO}$, and similarly $\mathcal{C}(\mathbf{y})$. The $\mathrm{O}$ atom at $\mathbf{x}$ remains (reacts) if $\mathcal{C}(\mathbf{x})$ is empty (nonempty), and similarly for the atom at $\mathbf{y}$.

At a threshold value of $Y, y_{2}(\simeq 0.5256$ on the square lattice), the ZGB model is said to exhibit "CO-poisoning." That is, the stationary state changes abruptly from an active one $\left(Y<y_{2}\right)$, with continuous production of $\mathrm{CO}_{2}$, to an absorbing state with all sites occupied by CO. (With all sites blocked, there is no way for $\mathrm{O}_{2}$ to adsorb.) The transition is sharply discontinuous. Experiments on surface-catalyzed $\mathrm{CO}+$ $1 / 2 \mathrm{O}_{2} \rightarrow \mathrm{CO}$ reactions do show a discontinuous transition between states of high and low reactivity as the partial pressure $p_{C O}$ (analogous to $Y$ in the model), is increased [59]. With increasing temperature, the transition softens, and at a certain temperature becomes continuous, about which the reaction rate becomes a smooth function of $p_{C O}$. This appears to be the result of thermally-activated, nonreactive desorption of CO.

A similar sequence of alterations is observed if we include nonreactive desorption of $\mathrm{CO}$ at a certain rate, $k$, in the ZGB model. With $\mathrm{CO}$ desorption there is no longer a CO-poisoned state, but for small $k$ the discontinuous transition between low and high $\mathrm{CO}$ coverages persists. Above a critical desorption rate $k_{c} \simeq 0.0406$, the coverages (and the $\mathrm{CO}_{2}$ production rate) vary smoothly with $Y[60,61,62]$. Simulation results indicate that at the critical point of the $\mathrm{CO}$ transition, the critical exponent $\nu=1$, and the reduced fourth cumulant $u \simeq 0.61$, as would be expected for the twodimensional Ising model [62]. 


\section{Hysteresis in the Ising Model}

For our final example we come full circle to the Ising model, forced out of equilibrium, this time, not through a bias or conflicting dynamics, but by a time-dependent external field $h(t)$. Of principal interest is a periodically varying field, for example, $h(t)$ sinusoidal or a square wave, with

$$
\langle h\rangle \equiv \frac{1}{\mathcal{T}} \int_{0}^{\mathcal{T}} h(t) d t=0,
$$

where $\mathcal{T}$ is the period of oscillation. Well below the critical temperature, and at sufficiently low frequencies, we expect to observe a hysteresis loop in the magnetization-field plane. As we raise the frequency, the system will at some point be unable to follow the rapid variations of the field, and the magnetization $m(t)$ will remain close to one of its stationary values, i.e., $+m_{0}$ or $-m_{0}$. That is, for high frequencies we expect

$$
Q \equiv \frac{1}{\mathcal{T}} \int_{0}^{\mathcal{T}} m(t) d t \neq 0
$$

whereas at low frequencies $Q$ should be zero.

For a sinusoidal field, the time-dependent mean-field equation describing this system predicts a continuous transition between a dynamically disordered $(Q=0)$ and a dynamically ordered $(Q \neq 0)$ state at a critical frequency, which depends on the temperature and $h_{0}=\max [h(t)]$ [63]. The transition was confirmed in various Monte Carlo simulations, and may be relevant to experiments on thin magnetic films [64, 65].

Rikvold and coworkers suggested that the transition between dynamically ordered and disordered states occurs when the period $\mathcal{T}$ becomes comparable to the metastable lifetime $\tau\left(h_{0}, T\right)$ of the state with the "wrong" sign of the magnetization (in a static field of magnitude $h_{0}$ ), and presented extensive simulation results to support this proposal [66]. These authors made a detailed numerical study of the critical behavior of $Q$ in two dimensions; their results for exponent ratios and the fourth cumulant are consistent with Ising values. While the very general symmetry arguments outlined above again favor such a conclusion, finding a path from the microscopic dynamics of this nonstationary process to an equation of the form of Eq. (7) seems a particularly challenging theoretical task.

\section{Summary}

I have reviewed a number of examples in which critical behavior in the Ising universality class appears, despite the presence of long-range interactions, lack of a temperature scale, or lack of equilibrium. The OrnsteinZernike approach that comes closest to reproducing Ising-like behavior was also reviewed. A general conclusion is that symmetries - of the order parameter and of the dynamics - are essential to determining critical behavior; the question of equilibrium is of minimal, if any, significance. On the other hand, we have seen that the effective, coarse-grained dynamics can look quite different from the microscopic interactions. In many cases there remains a considerable gap between these two levels of description.

\section{Acknowledgements}

It is a pleasure to thank George Stell and Tânia Tomé for collaborations that led to some of the results described in this work. This work was supported by $\mathrm{CNPq}$, Fapemig and MCT.

\section{References}

[1] L. S. Ornstein and F. Zernike, Proc. Acad. Sci. Amsterdam 17, 793 (1914).

[2] J. P. Hansen and I. R. McDonald, Theory of Simple Liquids, 2nd ed. Academic Press, London, 1986.

[3] R. Balescu, Equilibrium and Nonequilibrium Statistical Mechanics, Wiley, New York, 1975.

[4] Note that $c(r)$ plays a role analogous to a two-point vertex function in field theory.

[5] J. S. Høye and G. Stell, J. Chem. Phys. 67, 439 (1977); Molec. Phys. 52, 1057, 1071 (1984); Int. J. Thermophys. 6, 561 (1985).

[6] R. Dickman and G. Stell, Phys. Rev. Lett. 77, 996 (1996).

[7] D. Pini, G.. Stell, and R. Dickman, Phys. Rev. E 57, 2862 (1998).

[8] C. F. Baillie et al., Phys. Rev. B 45, 10438 (1992).

[9] M. F. Sykes et al., J. Phys. A: Math. Gen. 5, 640 (1972).

[10] A. J. Liu and M. E. Fisher, Physica A 156, 35 (1989).

[11] D. Pini, G. Stell, and N. Wilding, Mol. Phys. 95, 483 (1998).

[12] E. Kierlik, M. L. Rosinberg, and G. Tarjus, J. Stat. Phys. 89, 215 (1997).

[13] R. R. Singh and K. S. Pitzer, J. Chem. Phys. 92, 6775 (1990).

[14] M. L. Japas and J. M. H. Levelt Sengers, J. Phys. Chem. 94, 5361 (1990).

[15] T. Narayan and K. S. Pitzer, J. Phys. Chem 98, 9170 (1994); Phys. Rev. Lett. 73, 3002 (1994).

[16] P. P. Debye and F. Hückel, Phys. Z. 24, 185 (1923).

[17] G. Stell, K. C. Wu, and B. Larsen, Phys. Rev. Lett. 37, 1369 (1976).

[18] J. M. Caillol, D. Levesque, and J. J. Weis, J. Chem. Phys. 107, 1565 (1997).

[19] J. P. Valleau, and G. Torrie, J. Chem. Phys. 108, 5169 (1998).

[20] G. Orkoulas, and A. Z. Panagiotopoulos, J. Chem. Phys. 101, 1452 (1994); ibid., 110, 1581 (1999). 
[21] B. Hafskjøld and G. Stell, in Studies in Statistical Mechanics Vol. III, E. W. Montroll and J. L. Lebowitz, eds., North-Holland, Amsterdam, 1982.

[22] G. Stell, Phys. Rev. A 45, 7628 (1992).

[23] M. E. Fisher, J. Stat. Phys. 75, 1 (1994).

[24] Y. Levin and M. E. Fisher, Physica A 225, 164 (1996).

[25] A. Ciach and G. Stell, J. Molec. Liquids, to appear. e-print: cond-mat/0007286.

[26] R. Dickman and G. Stell, in Simulation and Theory of Electrostatic Interactions in Solution, L. R. Pratt and G. Hummer, eds., AIP Conference Proceedings 492, Melville, NY, 1999.

[27] A. Z. Panagiotopoulos and S. K. Kumar, Phys. Rev. Lett. 83, 2981 (1999).

[28] J. Frölich, R. Israel, E. Lieb, and B. Simon, J. Stat. Phys. 22, 296 (1980).

[29] B. Widom and J.S. Rowlinson, J. Chem. Phys. 52, 1670 (1970).

[30] J. S. Rowlinson and B. Widom, Molecular Theory of Capillarity, Clarendon Press, Oxford, 1982.

[31] E. Helfand and F. H. Stillinger, J. Chem. Phys. 49, 1232 (1968).

[32] R. Dickman and G. Stell, J. Chem. Phys. 102, 8674 (1995).

[33] M. E. Fisher, Phys. Rev. 176, 257 (1968).

[34] S.-N. Lai and M. E. Fisher, J. Chem. Phys. 103, 8144 (1995).

[35] R. Sun, H. Gould, J. Machta, and L. Chayes, e-print: cond-mat/0003516.

[36] D. S. Gaunt and M. E. Fisher, J. Chem. Phys. 43, 2840 (1965).

[37] D. S. Gaunt, J. Chem. Phys. 46, 3237 (1967).

[38] L. K. Runnels and L. L. Combs, J. Chem. Phys. 45, $2482(1966)$

[39] W. Kinzel, Z. Phys. B58, 229 (1985).

[40] See Braz. J. Phys. 30, no. 1 (March, 2000) for a collection of articles on this subject.

[41] R. Dickman, in Nonequilibrium Statistical Mechanics in One Dimension V. Privman, ed., Cambridge University Press, Cambridge 1996.

[42] G. Grinstein and M. A. Muñoz, in Fourth Granada Lectures in Computational Physics, Ed. P. Garrido and J. Marro, Lecture Notes in Physics, 493, 223 SpringerVerlag, Berlin, 1997.

[43] J. Marro and R. Dickman, Nonequilibrium Phase Transitions in Lattice Models Cambridge University Press, Cambridge, 1999.
[44] T. M. Liggett, Interacting Particle Systems, SpringerVerlag, (New York, 1985).

[45] J. W. Essam, J. Phys. A 22, 4927 (1989).

[46] R. Dickman and A. Yu. Tretyakov, Phys. Rev. E 52, 3218 (1995).

[47] M. J. de Oliveira, J. Stat. Phys. 66, 273 (1992).

[48] M. J. de Oliveira, J. F. F. Mendes and M. A. Santos, J. Phys. A 26, 2317 (1993).

[49] M. A. Santos and S. Teixeira, J. Stat. Phys. 78, 963 (1995).

[50] T. Tomé and J. R. Drugowich de Felício, Phys. Rev. E 53, 3976 (1996).

[51] P. L. Garrido, A. Labarta, and J. Marro, J. Stat. Phys. 49, 551 (1987).

[52] T. Tomé and M. J. de Oliveira, Phys. Rev. E 58, 4242 (1998).

[53] G. Grinstein, C. Jayaprakash, and Y. He, Phys. Rev. Lett. 55, 2527 (1985).

[54] A. Brunstein and T. Tomé, Phys. Rev. E 60, 3666 (1999).

[55] T. Tomé, Braz. J. Phys. 30, 152 (2000).

[56] B. Schmittmann and R. K. P. Zia, Statistical Mechanics of Driven Diffusive Systems, vol. 17 of Phase Transitions and Critical Phenomena, C. Domb and J. L. Lebowits. eds., Academic Press, New York, 1995.

[57] R. M. Ziff, E. Gulari, and Y. Barshad, Phys. Rev. Lett. 56, 2553 (1986).

[58] G. Grinstein, D.-W. Lai, and D. A. Browne, Phys. Rev. A 40, 4820 (1989).

[59] M. Ehsasi et al., J. Chem. Phys. 91, 4949 (1989).

[60] B. J. Brosilow and R. M. Ziff, Phys. Rev. A 46, 4534 (1992).

[61] J. W. Evans, J. Chem. Phys. 97, 572 (1992).

[62] T. Tomé and R. Dickman, Phys. Rev. E 47, 948 (1993).

[63] T. Tomé and M. J. de Oliveira, Phys. Rev. A 41, 4251 (1990).

[64] M. Acharyya and B. K. Chakrabarti, in Annual Reviews of Computational Physics, v. 1, D. Stauffer, ed., World Scientific, Singapore, 1994.

[65] B. K. Chakrabarti and M. Acharyya, Rev. Mod. Phys. 71, 847 (1999).

[66] P. A. Rikvold et al., in Computer Simulation Studies in Condensed-Matter Physics XII, D. P. Landau, S. P. Lewis, and H.-B. Schüttler, eds., Springer-Verlag, Berlin, 2000, and references therein. 